\title{
RESPUESTAS A LAS PARADOJAS SOBRE LA OMNIPOTENCIA DE DIOS
}

\author{
JUAN CAMILO HERNÁNDEZ RODRÍGUEZ \\ Universidad Pedagógica Nacional, Colombia
}

\begin{abstract}
RESUMEN: Podría decirse que uno de los debates que ha generado más discusiones, reflexiones y producciones al respecto en todas las corrientes de la filosofía (clásica, continental y analítica) ha sido sobre la existencia, naturaleza e influencia de Dios en el mundo. En el presente artículo se expondrán cinco paradojas planteadas por diversos filósofos (algunas de ellas modificadas y mejoradas) que abrirían la posibilidad de negar las perfecciones de Dios desde su naturaleza: su omnipotencia y su bondad. Una vez planteado cada problema, se expondrán las posibles soluciones a cada uno, mostrando que estos parten de ambigüedades, contradicciones o incomprensiones de la teoría cristiana. Adicional a lo anterior, se mostrará cómo esas respuestas podrían solucionar problemas filosóficos relacionados a la necesidad, la libertad y la determinación.
\end{abstract}

PALABRAS CLAVE: necesidad; posibilidad; bien; existencia; comunicación.

\section{Responses to the paradoxes on the omnipotence of God}

ABSTRACT: It might be said that one of the debates that has generated the most discussion, reflections and productions in this regard, in all the currents of philosophy (classic, continental and analytical), has been on the existence, nature and influence of God in the world. In the present article, five paradoxes raised by various philosophers (some of them have been adapted and improved) that would open the possibility of denying the perfections of God from his nature: his omnipotence and his kindness will be presented.

As soon as every problem was raised, the possible solutions will be presented to each one, showing that they are based on ambiguities, inconsistencies or misunderstandings of the Christian theory. In addition to the above, it will be shown how these responses could solve philosophical problems related to the need, freedom and self-determination.

KEY WORDS: necessity; possibility; wellbeing; existence; communication.

En en este artículo me dedicaré a responder cinco paradojas expuestas en contra de la omnipotencia de Dios. Haré esto con el fin de ampliar más el debate entre metafísicos teístas y los no teístas (no necesariamente creyentes) sobre la posibilidad de la existencia de un ser omnipotente que sea entendido como causa primera o primer principio de la existencia del mundo. Así, el plano en el que desarrollaré esta discusión es puramente argumental y no religioso; y, por congruencia con lo anterior, la única tesis que puedo sostener - con el fin de no tomar partido por algún bando y sesgar el análisis- sería de orden metametafísico. La tesis es la siguiente: para afirmar o negar la existencia de este ser con las características ya mencionadas ambos bandos deberán afirmar: por un lado, que es imposible que Dios, como ser omnipotente, exista $(\sim \vee \exists x[(x=\mathrm{d}) \wedge \mathrm{O} x])$; o bien, que es necesario que Dios, como ser omnipotente, exista $(\exists x[(x=\mathrm{d}) \wedge \mathrm{O} x], \mathrm{o}$ bien $\sim \nabla \sim \exists x[(x=\mathrm{d}) \wedge \mathrm{O} x])$.

El método que utilizaré para realizar mi análisis será el método analítico, según el cual se realiza un «esclarecimiento de conceptos significativos y 
proposiciones» "; no obstante, su análisis no es aislado o "purista» (dicho en otros términos, aislados de cualquier uso o aplicación), sino que como lo afirma Ayer: «En un diccionario buscamos, principalmente, las que podrían llamarse definiciones explícitas; en la filosofía, definiciones en uso »². Así, pues, este análisis tendrá un carácter reconstructivo histórico con el fin de evaluar el uso que hacen los filósofos cristianos de los conceptos "ser omnipotente», «existir» y "ser libre»; con esto, dicho método tiene dos características: es lógico y es histórico.

Como último aspecto previo, entenderé «paradoja» en estos casos como una antinomia ${ }^{3}$, es decir, una contradicción lógica entre los términos, principios, leyes o definiciones con algunas de sus derivaciones en las que a pasar de haber una perfecta validez en la deducción se llega a una contradicción de tipo: $\{\mathrm{df} x$ : $\left.\varphi x \rightarrow\left[(\varphi x \wedge \sim \varphi x) \rightarrow^{\wedge}\right]\right\} \rightarrow \sim \diamond \exists x(\varphi x)$ (si por definición $x$ es $\varphi$ entonces $x$ es $\varphi$ y no es $\varphi$, luego hay contradicción; por lo tanto, no puede existir un $x$ tal que ese $x$ sea $\varphi)$. Por lo tanto, y en concordancia con lo dicho arriba, las paradojas de los detractores de la existencia metafísica de este ser omnipotente son planteadas con el fin de negar lógicamente la posibilidad de la existencia de Dios; y, aunque es bien cierto que "ser omnipotente» y «existir» son conceptos distintos, en el caso de la definición de «Dios», con negar alguno de sus atributos infinitos ya es posible negar —o, al menos, poner en duda-su existencia.

\section{INTRODUCCIÓN}

Desde hace varios siglos una paradoja ha sido planteada por las posturas ateístas para demostrar la imposibilidad de la omnipotencia de Dios. Grosso modo, está planteada así:

«[I] - O bien Dios puede crear una piedra que Él no puede levantar, o Él no puede crear una piedra que no puede levantar.

- Si Dios puede crear una piedra que no es capaz de levantar, entonces Dios no es omnipotente (dado que Él no puede levantar la piedra en cuestión).

- Si Dios no puede crear una piedra que Él no puede levantar, entonces Dios no es omnipotente (dado que Él no puede crear la piedra en cuestión).

- Por lo tanto, Dios no es omnipotente». ${ }^{4}$

Esta paradoja, antiquísima por demás, ha sido uno de los argumentos más frecuentes de los detractores del cristianismo y de aquellos que acepten una teoría de la libertad como un don o un regalo recibido de Dios o la naturaleza.

1 CARnAP, R., La superación de la metafísica por medio del análisis lógico del lenguaje, trad. Nicolás Molina, UNAM, México, D. F. 2009, p. 30.

2 Ayer, A. J., Lenguaje, verdad y lógica, trad. Marcial Suárez, Orbis, Barcelona 1984, p. 96.

3 Ferrater Mora, J. Diccionario de filosofía, tomo 2 (L-Z), Editorial Sudamericana, Buenos Aires 1975, pp. 365-367, voz «paradoja».

4 Cowan, J. L. "The Paradox of Omnipotence Revisited» in: Canadian Journal of Philosophy, $\mathrm{n}^{\circ}$. 3, vol. 3 (1974), doi: http://dx.doi.org/10.1080/00455091.1974.10716896, p. 435. 
Esto lleva a cuestionar: a) el concepto de «necesidad» y su relación con el de «libertad»; b) si es posible conocer a Dios y, en caso de que sea posible, qué tipo de conocimiento se puede tener de Él; c) en caso de aceptar una teoría creacionista, cuál es la relación entre el creador y las criaturas; d) si es preferible un determinismo coercitivo por parte de Dios o que el hombre sea libre; y e) la objetividad o subjetividad del mal y si el estatus de este último es ontológico o meramente moral ${ }^{5}$.

En el presente trabajo se pretenderá, primero, exponer las objeciones; luego, se mostrará cómo el cristianismo - en especial, la propuesta de santo Tomás de Aquino-soluciona estos problemas antiguos y contemporáneos a partir del acto creador y de gobernanza del mundo de Dios, y cómo esta alternativa permite, si no solucionar el problema de manera definitiva, al menos sí comprender de mejor manera conceptos claves en la discusión como: «bien», «omnipotencia», «necesidad», «libertad», «creación» y «comunicación», «poder», «posible» etc.; y, por último, se brindará un análisis propio sobre las objeciones y la respuestas para evaluar qué implicaciones tiene cada una y cómo complementar algunas de las respuestas de Tomás con algunos presupuestos y teorías más actuales.

Como se ha mencionado, viarios han sido los argumentos planteados para demostrar la inexistencia de Dios, entre ellos, el principal ha sido sobre la omnipotencia de $\operatorname{Dios}^{6}$, y de estos se derivan: la maldad en el mundo, la libertad y el determinismo y la autodeterminación divina. Ante esto, autores cristianos y no cristianos han buscado comprender qué conceptos de fondo hay en dichas paradojas presentadas, encontrando en ellas los siguientes conceptos claves en todas: a) la necesidad, b) la libertad y c) el bien.

5 Es preciso aclarar que estos problemas no son las paradojas mismas, sino que son los cuestionamientos a los que llevan las mismas paradojas. Así, es posible que una paradoja tenga más de un problema y viceversa.

6 Solo se tomarán los principales argumentos relacionados con la omnipotencia y la libertad. Las respuestas como: «- No hay prueba de que existe... Así que, por lo tanto, usted ha concluido que no existe. - Eso es. - Mmmm... ¡Usted no puede probar que no existe! No se te puede pedir que demuestres un negativo. Eso es una ley de la lógica». Rand, A., "Ayn Rand con Donahue» [entrevista], en: The Phil Donahue Show, New York 1979. Recuperado de: https://www.youtube.com/watch?v=5wgpi3DcmV4, mm. 4:31-4:45 (cfr. Russell, B., "Is There a God?» In: Slater, J. G. \& Köllner, P. The Collected Papers of Bertrand Russell (pp. 543-548). Vol. 11, Routledge, London 1952) no serán tomados en cuenta por ser falacias de inversión de la carga y ad ignorantiam. Tampoco se tendrán en cuenta refutaciones tales como «La física puede explicar mejor el origen del universo» DE LA TORRE, A. «¿Son necesarias las religiones?» en: El Debate [programa de televisión] Canal 7, Buenos Aires 2011. Recuperado de: https:// www.youtube.com/watch?v=7BjvbX4_m1A\&t=323s, mm. 6:37-7:00; pues esto no niega en manera alguna su imposibilidad, sino que explica otra vía —a su juicio, más fiable o creíblepara entender el origen de la materia. En síntesis, tanto para argumentar la existencia como la no existencia habrá que argumentar la posibilidad o imposibilidad de esta por sí misma, y no evadiendo la responsabilidad de la defensa del argumento. "A cada cosa hay que asignarle una causa o una razón, tanto de por qué existe como de por qué no existe». Spinoza, B., Ética demostrada según el orden geométrico, trad. Atilano Domínguez, Trotta, Madrid 2000, I, prop. 11/OP, 8 . 
Podría sintetizarse la consecuencia de las objeciones en la siguiente argumentación:

«[II] Dios es imposible, innecesario, indeseable y no hay ninguna evidencia de su existencia. El Dios de las religiones, omnipotente y de bondad infinita no puede existir. La existencia del mal implica una contradicción entre estas propiedades. Si existen niños que sufren por cáncer terminal no puede existir Dios. Existe Auchwitz, no existe Dios; dice Primo Levi [...] Dios es indeseable por las bestialidades hechas en su nombre.» ${ }^{7}$

Así, de estas paradojas (I y II) dos problemas emergen: la autodeterminación y la acción malvada de las criaturas. Podría decirse, entonces, que el problema del mal puede adecuarse así: a) Dios no puede crear el mal o algo mayor a él; luego, no es omnipotente; y b) Dios no puede controlar la maldad del hombre; luego, no es omnipotente o no es bueno. Puestas ya las objeciones, se procederá entonces a explicar cómo el cristianismo — sobre todo, santo Tomás de Aquino- logra sortear estos inconvenientes a partir de la teoría de la creación/ ex-sistencia.

\section{Respuestas de Tomás y el CRistianismo}

Podría decirse que el problema de los anteriores argumentos está en no comprender de manera central cuál es el tipo de naturaleza que posee Dios dentro de la teoría creacionista cristiana y cuál es el tipo de conocimiento que tenemos y podemos alcanzar de él.

¿Cuál es la definición que tenemos de Dios? Es el Ser que está siempre en acto $^{8}$. Al estar siempre en acto, posee todas las cualidades perfecciones posibles (omnisciencia, omnipotencia, omnipresencia, eternidad, etc.). Dado que todos los seres poseen una esencia determinada ${ }^{9}$, la del Ser que está siempre en acto debe ser un principio superior al Ser mismo, el cual le permite ser motor de

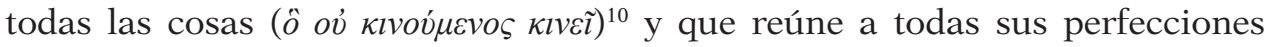
( $\dot{\varepsilon} v \varepsilon \varepsilon \lambda \dot{\eta} \chi \varepsilon \iota \alpha)$ : el bien ${ }^{11}$.

7 De la TORRe, o. c., mm. 6:06-7:53

8 Santo Tomás De Aquino, El ente y la esencia, trad. Manuel Fuentes Benot, Aguilar, Buenos Aires 1963, c. 4, § 33.

9 Entiéndase en este caso esencia como quididad, es decir, no como «[...] algo común a todas las naturalezas». De Aquino, ibíd., c. 1, § 4, sino como «[...] aquello por lo cual una cosa ha de ser tal cosa» ibíd., c. 1, § 5, pues, dado que Dios es Uno (cfr. De Aouino, S. T. Suma de teología, cuarta edición, Biblioteca de Autores Cristianos, Madrid 2001, I, q. 11, a. 3), no hay especie ni género posible para él.

10 Santo Tomás De Aouino, Del ente y la esencia, c. IV, § 34; S.T., I, q. 2, a. 3, sol.

11 Ibíd., I, q. 6, a. 3. Aquí se puede ver la fuerte influencia del neoplatonismo en Tomás al identificar al principio de todas las cosas con el Uno y relacionarlo con el Bien. $C f r$. Plotino, Enéadas, trad. Jesús Igal, tomo 3, Gredos, Madrid 2002, VI, 4-5; 9. Esta idea proviene de Platón, para quien «el Bien es superior al Ser». Cfr. Platón, La República, trad. José Pabón y 


\subsection{La «existencia» ${ }^{12}$ de Dios}

Según Tomás, la existencia de Dios no es evidente por sí misma ${ }^{13}$ — como creía Anselmo ${ }^{14}$ — , ya que «[...] lo que en cuanto tal es cognoscible en grado sumo, deja de ser cognoscible por algún entendimiento por sobrepasar su capacidad ${ }^{15}$; sino que solo se tiene una intuición de él «[...] Como, por ejemplo, saber que alguien viene no es saber que Pedro viene, aunque sea Pedro el que viene» ${ }^{16}$.

A pesar de esto, sí se puede demostrar su existencia ${ }^{17}$, pero de un modo distinto al que conocemos las cosas (por su causa [propter quid]): por los efectos $(q u i a)^{18}$ de esa causa primera, esto es: la creación. Así, Tomás ofrece cinco vías para demostrar su existencia: como motor inmóvil (dado que las cosas no se mueven por sí mismas, debe haber algo inmóvil que las mueva), como causa eficiente (si todas las cosas tienen una causa, debe haber una primera causa no

Manuel Fernández-Galiano, edición bilingüe, Centro de Estudios Políticos y Constitucionales, Madrid 2006, VI, 504c-509b; Sofista, en: Diálogos: Critón. Gorgias. Menón. Fedro. Sofista. Político. Cartas, edición bilingüe, Centro de Estudios Políticos y Constitucionales Madrid, 2013, 237d-238a; Parménides, trad. María Isabel Santa Cruz, en: Obra completa, tomo 2, Gredos, Biblioteca de Grandes Pensadores, Madrid 2014, 144b.

12 De hecho, hablar de la «existencia de Dios» es una imprecisión que llevaría a contradicciones o sinsentidos; pues - como lo indican FREGE, G., Los fundamentos de la aritmética, trad. Hugo Padilla, en: Escritos sobre lógica, semántica y filosofía de las matemáticas, UNAM, México, D. F. 2016, §§ 49-53, y KANT, I., [KrV] Crítica de la razón pura, trad. Mario Caimi, edición bilingüe, Fondo de la Cultura Económica, México, D. F. 2011, A597/B265- predicar de Dios su existencia es carente de contenido. Lo anterior se debe a que: primero, la existencia solo es aplicable a los entes o criaturas; segundo, porque, dado que «existencia» significa "estar fuera de su raíz», no tiene sentido aplicar este predicado a Dios. Así, el estado de Dios es la subsistencia, no la existencia. Más bien, este tipo de pruebas lo que indican es a partir de la existencia intuitivamente el Ser de Dios (véase 2.2.3).

13 Santo Tomás De Aouino Suma teológica, I, q. 2, a. 1.

14 San Anselmo, Proslogion, trad. Jordi Corominas y Judith Ribas, Tecnos, Madrid 1998 , c. II. A pesar de esto, argumentos un tanto más sólidos propuestos por Descartes, R. Meditaciones acerca de la Filosofía Primera. Trad. 1a ed. del latín por Jorge Aurelio Díaz, edición trilingüe, Universidad Nacional de Colombia, Bogotá 2014, VII 45; Discurso del método, trad. Manuel García Morente, en: Flórez Miguel, C. (ed.), Obra completa, Gredos, Biblioteca de Grandes Pensadores, Madrid 2011, AT VI, 35) Leibniz, G. W. Tratado de metafísica y Monadología, en: Echeverría, J. (ed.). Escritos filosóficos. Gredos, Biblioteca de grandes pensadores, Madrid: 2011, según los cuales la idea de Dios es mucho más perfecta y abarcativa que el entendimiento y, por lo tanto, debe existir Dios. A partir de esto Spinoza formuló el siguiente argumento modal, siendo este mucho más refinado que el de sus congéneres: «[...] existe necesariamente aquello de lo que no se da razón ni causa alguna que impida que exista. Así pues, si no puede darse causa o razón alguna que impida que Dios exista o que le prive de su existencia, habrá que concluir, absolutamente, que existe de un modo necesario». SPINOZA, B., Ética., prop. 11/OP, 8-9; es decir, "para todo $x$, si no es posible que ese $x$ no exista, entonces ese $x$ existe necesariamente»: $\forall x(\sim \vee \sim \exists x \rightarrow ` \exists x)$.

15 Santo Tomás De Aquino S. T., I, q. 2, a. 1, sol.

16 Ibid., I, q. 2, a. 1, ad. 1.

$17 \quad$ Ibid., I, q. 2, a. 2.

18 Ibíd., I, q. 2, a. 2, sol; Еsсото J. D., Cuestiones cuodlibetales, trad. Félix Alluntis, Biblioteca de Autores Cristianos, Madrid 1968, q. VII, $a$. I, 1, § 7. 
causada), como ser necesario (dado que lo material es contingente, debe existir un ser necesario del cual proceda todo), como ser supremo/perfecto (a todo ser imperfecto se le antepone lo perfecto, esto último es Dios) y como ordenador de las cosas (dado que la naturaleza parece tener algún orden, es necesario que este orden provenga de un intelecto perfecto, esto es: Dios) ${ }^{19}$.

Este tipo de demostraciones, según Tomás, no son contrarias a la razón, pero tampoco pueden prescindir de la fe ${ }^{20}$. Esto se debe a que «[...] la esencia divina no puede ser vista con los ojos corporales ${ }^{21}$, y, dado que su naturaleza sobrepasa nuestra capacidad, es necesario superar la multiplicidad de la ciencia ${ }^{22}$, pues sigue manteniéndose una doble condición: el ser pensado y el ser que lo piensa ${ }^{23}$. Con esto, es necesario que al entendimiento humano «[...] se le prepare con alguna disposición que esté por encima de la naturaleza ${ }^{24}$. Además, es necesario recibir esta disposición de un ser en acto, para elevar al entendimiento todavía en potencia y unirse ${ }^{25}$ al acto perfecto, que es Dios. A

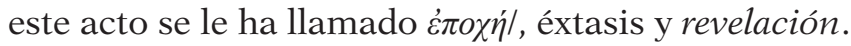

«La perfección consiste en que los inteligibles le son dados en acto, en una intuición que los representa en el espíritu, y es "luz sobre luz" ${ }^{26}$. Y la facultad consiste en que le pertenece realizar el inteligible adquirido, llevado así a su culminación, como es el objeto de la intención, desde el momento en que ella lo quiere, sin necesidad de adquirirlo [en ese último momento], y es la lámpara. Esa perfección se llama inteligencia adquirida, y esa facultad, inteligencia en acto. ${ }^{27}$

Así, pues, se entiende que, aunque este tipo de conocimiento que tenemos no

19 Santo Tomás De Aquino, S. T., q. 2, a. 3, sol. No se hará mucho énfasis en la explicación de estas pruebas, pues: primero, es uno de los temas más explicados y debatidos de Tomás; y segundo, el presente ensayo pretende centrarse en las respuestas los argumentos en contra de la omnipotencia divina. Grosso modo, las cinco vías se basan en hipótesis que surgen de la contraposición de la imperfección y limitación de la naturaleza. Téngase en cuenta que la validez de estas vías es bastante débil y, por ello, no tienen valor de necesidad, sino que es intuitivo y, más que ser necesario, es de plausibilidad.

20 Ibíd., II-II, qq. 1-16; Averroes, «Doctrina decisiva y fundamento de la concordia entre la revelación y la ciencia», en: FERNÁNDEZ, C. (ed.), Los filósofos medievales (pp. 618-631 [§§ 1053-1086]), tomo 1, Biblioteca de Autores Cristianos, Madrid 1979, § 1.249.

21 Santo Tomás De Aouino, S. T., I, q. 12, a. 3.

22 SAn Agustín, «De la cuantidad del alma», en: Obras filosóficas, trad. Eusebio Cuevas, ed. bilingüe, Biblioteca de Autores Cristianos, Madrid 1947, VII, § 12.

23 Plotino, o. C., VI, 4.

24 Santo Tomás De Aquino, S. T., I, q. 12, a. 5, sol.

25 Entiéndase esta unión no como una relación de participación necesaria entre Dios y el hombre, sino el encuentro entre la criatura con Él por una relación de comprensión de su esencia; $c f r$. Plotino, o. c., VI, 9. En el caso de Agustín, una relación de conocimiento interior; SAN Agustín, Confesiones, trad. Pedro Rodríguez de Santidrián, Altaya, Barcelona 1993, cc. XI-XIII.

26 También está en Sal, 35, 10: «Con tu luz, veremos la luz». Cfr. SAnto Tomás De Aouino, o. c., I, q. 12, a. 6, sol.

27 Avicena, «Libro de los teoremas y avisos», en: Fernández, C. (ed.), Los filósofos medievales (pp. 618-631 [§§ 1053-1086]), tomo 1, Biblioteca de Autores Cristianos, Madrid 1979, § 1.059. 
es igual al de la ciencia, sí es racional; solo que requiere de creer para entender y entender para creer ${ }^{28}$.

\subsection{La necesidad (en sí y por relación) en la acción divina}

De lo anteriormente dicho se deriva lo siguiente: comprender la esencia y propiedades de Dios solo es posible si la naturaleza divina se le revela al entendimiento humano; además, "a cualquier entendimiento creado le resulta imposible comprehender a Dios ${ }^{29}$. De esto, resulta la primera respuesta a la paradoja I es un tanto obvia: la esencia de Dios y sus perfecciones no se pueden conocer de la misma manera que las justificaciones sobre la potencia de los hombres o los animales, pues la única forma de demostrar la existencia y conocer la naturaleza divina es quia (por los efectos) y no propter quid (por las causas).

Parece que en I Cowan no solo confunde el tipo de conocimiento de la naturaleza divina, sino que también confunde dos tipos de necesidad.

«Necesario se dice en dos sentidos ${ }^{30}$ : absolutamente o según lo supuesto. Se juzga como absolutamente necesario por el contenido de los términos: bien porque el predicado está en la definición del sujeto, como, por ejemplo, es necesario en el hombre ser animal; bien porque el sujeto pertenece a la razón del predicado, como, por ejemplo, es necesario que un número sea par o impar. Por otra parte, no es necesario, por ejemplo, en Sócrates estar sentado. No es absolutamente necesario, pero sí puede decirse necesario según lo supuesto. Ejemplo: suponiendo que esté sentado, es necesario que esté sentado mientras está sentado.» ${ }^{31}$

Así, pues, la primera paradoja ya es desechada; se están confundiendo dos tipos de necesidades: la intrínseca (ad intra) y la extrínseca (ad extra) $)^{32}$, donde la primera es más fuerte (esencial) que la otra (accidental) ${ }^{33}$; pues las potencias de Dios subsisten en él necesariamente de manera eterna, mientras que los actos divinos no le son necesarios para su ser, entre ellos, la creación.

28 SAN ANSElmo, o. c., c. I.

29 Santo Tomás De Aquino, S. T., I, q. 12, a. 7, sol.

30 «En cuanto al Ser Necesario, o bien es necesario por sí o no es necesario por sí. El que es necesario por sí es el que es la causa de su propia esencia, no a causa de alguna otra, sea ésta la que fuere, pues la hipótesis de su no-existencia entrañaría una contradicción. En cuanto al ser necesario que no lo es por sí, es el que se hace necesario por alguna cosa distinta de él que le es agregada; por ejemplo, cuatro es necesario no por sí, sino en tanto que dos y dos son agregados; la combustión no es necesaria por sí misma, sino en cuanto se presupone el concurso de la potencia activa por naturaleza, es decir, de lo que hace arder y de lo que es quemado». Avicena, Sobre metafísica, trad. Miguel Cruz Hernández, Revista de Occidente, Madrid 1950, 1950, § 14/ p. 115; cfr. ARIstóteles, Metafísica, trad. Valentín García Yebra, edición trilingüe. Gredos, Madrid 2014, $\Delta$, c. 5, 1015a20-35.

31 Santo Tomás De Aquino, S. T., I, q. 19, a. 3, sol.

32 Еsсото, о. с., q. 7, § 1.

33 Cfr.: Hoffman, J. y Rosenkrantz, G. S., «Omnipotence Redux» en: Philosophy and Phenomenological Research, n. 49 (1988), recuperado de: https://www.jstor.org/stable/ 2107977?seq=1\#page_scan_tab_contents. 


\subsubsection{La necesidad en la creación}

Se dice que la creación no es necesaria a Dios porque el acto de creación es: a) es necesidad ad extra, no ad intra. Así, lo único necesario en su acto creador es que para que se le llame creador es necesario que haya creado algo (el mundo), pero no le era necesario a Dios crear el mundo para ser Dios; en otras palabras, Dios pudo no haber creado el mundo y seguir «existiendo», solo que ya no como un Ser creador $^{34}$.

Así, dado que el acto creador de Dios es necesario, es preciso que este tipo de relación sea libre, de comunicación. Es decir, a Dios, «[...] no le corresponde actuar para adquirir algún fin, sino que tan sólo intenta comunicar su perfección, que es su bondad $»^{35}$. Así, a la voluntad de Dios solo le corresponde querer el bien. «[...] La voluntad divina tiene una relación necesaria con su bondad, que es su objeto propio. Por lo tanto, Dios quiere por necesidad su bondad $»^{36}$.

No obstante, alguien podría objetar una «tercera paradoja» (III), la cual diría: Si Dios es omnipotente puede hacer algo mal/el mal ${ }^{37}$. Dios no puede hacer algo mal/el mal. Por lo tanto, no es omnipotente.

Lo anterior se demuestra, entre otras cosas, porque, teniendo en cuenta que la esencia de Dios es su bondad, la maldad no le pertenece, sino que tendría ser ajeno a él. Así, hacer el mal no es un poder, sino una debilidad.

«En efecto, quien puede hacer todo esto puede hacer lo que no le conviene y lo que no debe. Cuanto más puede hacer esto, tanto más poder tienen sobre la adversidad y la perversidad, y él menos contra ellas. Así pues, quien tiene ese poder, no lo tiene por su potencia, sino por su impotencia. Si se dice que tiene poder por esto, no se dice realmente que tenga poder él mismo, sino que su impotencia hace que otro tenga poder sobre él.» ${ }^{38}$

34 Esto quiere decir que el acto del ser de Dios es interno, autosuficiente y que no depende de su comunicación en sentido alguno; es decir, es necesidad per se y no por relación a algo (véase la nota al pie 30).

35 Santo Tomás De Aquino, $S$. . T. I, $q$. 44, a. 4, sol; $c f r$. Pseudo-Dionisio, Pseudo-Dionisio Aeropagita, «De los nombres divinos», en: Fernández, C. (ed.), Los filósofos medievales (pp. 496-520 [§§ 787-837]), tomo 1, Biblioteca de Autores Cristianos, Madrid 1979, c. IV, § 1.

36 Santo Tomás De Aouino, S. T., I, q. 44, a. 4, sol. Esto se sigue necesariamente si, tal como es asumido ontológicamente el bien en el neoplatonismo, el mal no es más que una degradación del Bien; por lo tanto, el mal, en sentido ontológico, es imperfección. Cfr. PloTino, o. c., VI, 1-9; SAN Aguistín, «De la naturaleza del bien: contra los maniqueos», en: Obras filosóficas, trad. Mateo Lanseros, Biblioteca de Autores Cristianos, Madrid 1947, c. IV.

37 Caso similar pasa con la piedra que Él mismo no puede levantar; pues no poder crear algo que niegue su omnipotencia no indica impotencia, sino lo contrario, reafirma su omnipotencia; ya que «El Ser Necesario es el ser que sería contradictorio suponer como no existente». AvicenA, o. c., § 14/ p. 114 (cfr. LeiBniz, G. W., La profesión de fe del filósofo, trad. Francisco de P. Saramanch, Aguilar, Buenos Aires 1966, p. 58); o, dicho de otro modo, «[...] [es aquello]» que no podría haber sido de otra manera». KRIPKE, S., Identidad y necesidad, trad. Margarita M. Valdés, UNAM, México, D. F. 1978, pp. 26-27; es decir, si es necesario que Dios sea omnipotente, entonces su imposibilidad implica contradicción, así: $(\mathrm{O} d>\diamond \sim \mathrm{O} d) \rightarrow$; o también, que Dios necesariamente sea omnipotente es igual a decir que no es posible que no sea omnipotente: $\mathrm{O} d=\sim \nabla \sim \mathrm{O} d)$.

38 San Anselmo, o. c., c. VII. 
De esto se sigue que la imposibilidad del mal como creación divina o de imperfección en el acto creador no niega la omnipotencia divina, sino que la reafirma. Así pues, la respuesta a la paradoja de la imposibilidad de Dios de hacer el mal o crear algo más perfecto que él ${ }^{39}$ es esta: $\alpha$ ). La paradoja I carece de sentido, pues confunde dos maneras de decir «necesario»; $\beta$ ). Suponiendo que no existiese esa confusión, la analogía de la roca con la potencia de Dios confunde los dos modos de potencia, siendo una (la creación de la piedra) un conocimiento demostrado quia y la otra (la potencia de levantarla) un conocimiento propter quid. Por lo tanto, la crítica tampoco tiene cabida; y $\gamma$ ). Suponiendo que $\alpha$ y $\beta$ no se diesen, es contradictoria en sí misma la refutación, pues lo que I exige es que se haga posible lo imposible para reafirmar algo, cosa absurda de entrada; o algo es posible o es imposible, pero no puede ser posible e imposible e imposible a la vez, pues esto lleva a una contradicción: $[(\diamond \mathrm{P} \wedge \sim\rangle \mathrm{P}) \rightarrow] \rightarrow(\diamond \mathrm{P} \vee \sim \diamond \mathrm{P})$.

Por otra parte, la paradoja $\mathbf{I I I}^{40}$ parece caer por su propio peso; pues: $\alpha$ ). pide al ser perfecto que obre de manera imperfecta; y $\beta$ ) dado que la esencia de Dios es la bondad y actuar mal es apartarse del Bien (que es Dios) ${ }^{41}$ entonces pedirle a Dios que se aleje de sí mismo o de su esencia carece de sentido en lo absoluto $^{42}$. Si Dios es el Bien y el mal es alejarse de Dios, pedirle a Dios que se aleje de sí mismo es un sinsentido. Con esto la siguiente afirmación de Agustín no es un problema, sino que, por el contrario, reafirma la omnipotencia divina: «Siendo Dios bueno, como tú sabes o crees-y ciertamente no es lícito creer lo contrario-, es claro que no puede hacer el mal» ${ }^{43}$.

\subsubsection{La omnipotencia sobre lo posible}

Ante lo anterior se puede decir siguiente: $\alpha$ ). al decir que Dios es omnipotente, solo se indica que Él puede hacer absolutamente solo lo que es posible (principio lógico básico $\left.{ }^{44}\right)$ :

39 Como ya se ha indicado más arriba, el término «bien» es tomado de la teoría

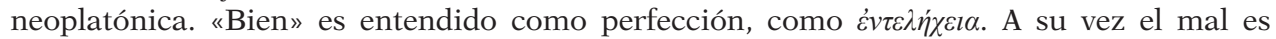
entendido por el neoplatonismo y por gran parte del cristianismo como imperfección o degradación del bien.

40 Dado que la paradoja II introduce elementos morales, se responderá en 2.3.

41 SAn Agustín, «Del libre albedrío», trad. Evaristo Seijas, en: Obras filosóficas. Tomo 3, Biblioteca de Autores Cristianos, Madrid 1947, I, 1, § 2.

42 «[... Aunque tal vez Dios no haya querido que me engañe de esa manera, porque se dice que es sumamente bueno; pero si contradijera a su bondad le sería ajeno el permitir que me equivoque a veces; y esto último sin embargo no se puede decir». Descartes, Meditaciones, AT, VII, 21.

43 San Agustín, o. c., I, 1, § 1.

44 Aunque el argumento es, evidentemente, tautológico (Dios solo puede hacer todo lo que se puede hacer/si algo es posible para Dios, entonces debe ser posible), sí permite ver los errores de los argumentos anteriores, que, antes que mostrar impotencia en Dios al no poder Él hacer imposibles, son solo refutaciones absurdas; pues la omnipotencia «[...] es potencia respecto de lo posible, entendido, no en general, en cuando se opone a lo imposible, 
«Por regla general todos confiesan que Dios es omnipotente. Pero parece difícil determinar a razón de omnipotencia. Pues cabe la duda en la afirmación: Dios todo lo puede. Pero, pensándolo correctamente, como el poder se refiere a lo posible, al decir que Dios todo lo puede, o más correcto es entender que puede todo lo que es posible, y por eso es llamado omnipotente [...] Por lo tanto, todo lo que no implica contradicción cabe dentro de los posibles respecto a los que Dios es llamado omnipotente» ${ }^{45}$

y $\beta$ ). los principios necesarios no pueden ser violados por Dios, pues, dado que lo necesario es aquello que no puede ser no posible, sucede lo mismo que en $\alpha$ ); Dios solo puede hacer lo que es posible (claro está, si efectivamente es algo necesario y no solo algo que se cree que es necesario, pero que no lo es). Dice santo Tomás:

«Los principios de algunas ciencias como la lógica, la geometría y la aritmética, se toman solamente de los principios formales de las cosas, de los que dependen esencialmente. Por consiguiente Dios no puede hacer lo contrario a dichos principios; como que el género no sea predicable de la especie, o que los radios de una circunferencia no sean iguales, o que el triángulo rectilíneo no tenga tres ángulos iguales a dos rectos. ${ }^{46}$

De lo anterior se deduce, pues, que la imposibilidad de negar Dios lo necesario o de hacer lo imposible son objeciones que atenten contra la omnipotencia divina, sino que, dada la definición de «necesario» y de «imposible», la omnipotencia solo indica que se Dios tiene todas las potencias, el poder de hacer todo lo posible. En caso de que Dios pueda hacer algo imposible, entonces eso perdería su condición de imposible y se convertiría en posible (al menos para Dios $)^{47}$.

ni en cuanto posible se convierte con lo producible o e cuanto se opone a lo absolutamente necesario por sí, sino respecto de posible en cuando se identifica con lo causable». Escoto, o. c., $q$. VI, $a .1,2, \S 8$. De lo anterior se infiere que la omnipotencia por relación evita la paradoja propuesta, pues se sustenta en una necesidad por relación y no necesidad por sí; expuestas por Geach («Omnipotence». Philosophy, n. 48, vol. 183, 1973. doi: http://dx.doi.org/10.1017/ S0031819100060381) como los sentidos 2 a 4 de «omnipotencia». Por otra parte, si se tomase a la omnipotencia ad intra, no habría contradicción alguna, pues estarían confundiéndose dos sentidos distintos: necesario por sí $(\square \mathrm{O} d)$ y necesario por relación/supuesto $(\diamond \phi d \rightarrow \psi d)$. «Es verdad que todo lo que tiene que ser, será verdaderamente, pero no necesariamente, en virtud de una necesidad absoluta, es decir, sea lo que sea lo hagas o dejes de hacer, Pues el efecto no es necesario más que a partir de la hipótesis de la causa» (LeIBNIz, Profesión de fe del filósofo, p. 61).

45 Santo Tomás De Aouino, S. T., I, q. 25, a. 3, sol.

46 Santo Tomás De Aquino, S. T., Suma contra los gentiles, trad. Carlos Ignacio González, Editorial Porrúa, México, D. F. 1998, II, c. 25, § 4.

47 Por otra parte, si alguien afirmase que es lícito afirmar ontológicamente predicados de Dios aunque atenten contra la lógica porque la naturaleza divina puede exceder los límites de nuestro modo de pensar (DEscarTes, o. c., AT, VII, 47) o de nuestro lenguaje (WITTGENSTEIN, Tractatus logico-philosophicus, trad. Jacobo Muñoz e Isidoro Reguera, en: Reguera, I. [ed.], Obra completa, vol. 1, edición bilingüe, Gredos, Biblioteca de Grandes Pensadores, Madrid $2009,6.432-6.522$ ), esto antes reafirmaría que cualquiera de las paradojas planteadas son en 


\subsubsection{La relación existencial creador-criatura: la comunicación del bien}

Con las explicaciones presentadas hasta el momento ya han sido clarificados los principios de la acción divina y sus tipos de necesidad; sin embargo, siguiendo lo prometido al inicio, todavía falta explicar lo siguiente: qué tipo de relación existencial hay entre las criaturas con el creador, cómo el hombre recibe de Dios el don de la libertad, cómo es posible el mal, cómo la libertad es un bien superior al determinismo y cómo se da el tránsito del es al debe en la teoría tomista. A partir de estas explicaciones se podrá responder al problema IV, planteado a continuación:

«(IV) — "Dios es la suma de toda realidad (ens realissimum)

— [...] Todo predicado positivo originario, todo predicado que, no siendo derivado de otros, exprese una realidad, le corresponde, por definición, al ens realissimum que en encierra en sí toda la realidad"48

- Por lo tanto, si se puede predicar de algo real que es contingente e imperfecto, lo mismo se puede predicar del todo o la suma (del ens realissimum).

— Las criaturas (entes reales) son contingentes e imperfectos; luego, Dios lo es. $»^{49}$

El anterior razonamiento parece ser mucho más coherente y sólido que los dos anteriores; sin embargo, el error está en admitir de entrada la definición de Dios como «[...] la suma de toda la realidad». Parte de ese error está en confundir el acto creador como una extensión de Dios o, dicho de otra forma, de participación.

Quizás, la razón de esta confusión está en la siguiente afirmación: «Entonces dijo Dios: Hagamos al hombre a nuestra imagen, conforme a nuestra semejanza» ${ }^{50} ; \mathrm{y}$, teniendo en cuenta que para crear algo es necesario tener una referencia, un $\pi \alpha \rho \alpha ́ \delta \varepsilon \imath \gamma \mu \alpha$, como lo indicó Platón ${ }^{51}$, dado que nada había sido

lo absoluto problema alguno para la naturaleza divina, y, por lo tanto, estas no presentarían ningún problema. Sin embargo, concuerdo con Mavrodes al creer que el significado de «omnipotencia» no exige exceder las leyes de la lógica, sino que basta con que este ser pueda hacer todo lo lógicamente posible para recibir tal denominación. Cfr.: MAvrodes, G. «Some Puzzles Concerning Omnipotence» en: The philosophical review, n. 72, vol. 2, 1963. Recuperado de: https://www.jstor.org/stable/2183106?seq=1\#page_scan_tab_contents.

48 Kant, parafraseado por Caimi en: KanT, o. c., p. LV.

49 En realidad, el argumento de Kant suele estar en este punto más orientado a la imposibilidad de conocer algo de Dios, basándose en que, dada su condición de absoluto, no se puede tener experiencia alguna con Él, (KANT, 2011 [KrV], A600/B628). Sin embargo, como se mostró en 2.1, hay dos "formas» para poder conocerle: por sus efectos (y estas pruebas [como las cinco vías de Tomás] si parten de la experiencia, aunque no capturan la esencia de Dios) y por sus causas (donde se puede conocer por medio de la revelación), que sí sería una experiencia, solo que en un sentido distinto: la experiencia de sentir a los propios sentidos como insuficientes para poder observar la luz divina (por exceso de luz).

50 Gen., 1, 26.

51 Platón, Timeo, trad. Francisco Lisi, en: Obra completa., tomo 3. Gredos, Biblioteca de Grandes Pensadores, Madrid 2014, 48e-49a. Es por eso que se tradujo al griego como

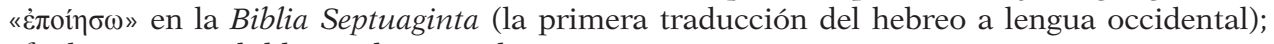
cfr.: https://www.bibliacatolica.com.br/septuaginta/genesis/1/. 
creado antes, entonces, al Dios ser el «modelo» de la creación, las criaturas participan de su naturaleza, en algún sentido. Además de lo anterior, puesto que «existir» no es un predicado genuino ${ }^{52}$ al igual que como lo es «ser» ${ }^{53}$, entonces afirmar la existencia de los entes de una manera independiente al Ser de Dios significa algo si, y solo si, se da una relación de participación entre el ente y Dios.

Ante esto, es preciso responder: primero, como se dijo en 2.2.1., el acto creador es un acto libre de Dios; pues la definición de Dios como ser absolutamente perfecto no implica necesariamente ser creador ${ }^{54}$. No obstante, tomando el segundo sentido de necesidad ${ }^{55}$, si se supone a Dios como creador, sí es necesario que cree el mundo para ser creador, mas no para ser. Segundo, basándonos en lo anterior, el acto creador de Dios es comunicativo ${ }^{56}$, es decir, es el acto mediante el cual el bien, de manera libre, se difunde en otros:

«Dios no sólo se quiere, sino que también quiere lo distinto a Él. Esto resulta claro por la comparación que antes se puso $(a$. 1). Pues una cosa natural no sólo tiene inclinación natural con respecto al propio bien, para conseguirlo si no lo tiene y para descansar en él si lo tiene; sino para difundir el propio bien en otros en la medida de lo posible. Por lo cual, vemos que todo agente, en cuanto está en acto y es perfecto, hace lo semejante a él. Por eso, en la razón de voluntad entra que el bien poseído se comunique a los demás en la medida de lo posible.» ${ }^{57}$

Así, pues, dada que su relación con la criatura no es una necesidad en el primer tipo (ad intra), sino en el segundo ( $a d$ extra), no es posible que la creación se dé por participación, pues la participación es una relación del todo con las partes (interna), mientras que la comunicación es una relación virtual (externa). Por otra parte, bien es cierto que la creación exige de un modelo o paradigma, pues la creación de Dios es ex nihilo ${ }^{58}$; así, el modelo o paradigma de la creación tiene que provenir de Dios mismo - y por eso se dice que las criaturas fueron hechas por Dios a su imagen y semejanza-, y esto es: sus formas ejemplares, es decir, sus ideas ${ }^{59}$.

$52 \quad$ KANT, o. c., A597/B265.

53 Aristóteles, Física, en: Candel, M. (ed.), Obra completa I, trad. Guillermo R. de Echandía, Gredos, Biblioteca de Grandes Pensadores, Madrid 2011, I, 3, $185 a 35$.

54 Como se dijo en la nota al pie 11, necesario es aquello cuya imposibilidad es imposible $(\square \mathrm{P}=\sim\rangle \sim \mathrm{P})$. Cuando se dice que el acto creador de Dios es libre lo que se indica es que es un acto posible (es decir, es posible que Dios no hubiera creado el mundo). Ahora bien, cuando se dice que es necesario que el acto creador sea por comunicación y no por participación, lo que se indica es que, suponiendo que se dé el acto creador, para que se dé es necesario que sea de esta primera forma y no de la segunda. Es decir, la primera condición es ad intra o necesidad por sí y la segunda ad extra o necesidad por relación.

55 Santo Tomás De Aquino, S. T., I, q. 19, a. 3, sol.

56 Pseudo-Dionisio, o.c., c. IV, $\S 1$.

57 Santo Tomás De Aquino, ibíd.

58 Santo Tomás De Aquino, S. T., I, q. 45, a. 1.

59 Santo Tomás De Aquino, S. T., I, q. 44, a. 3, sol. 
Sin embargo, de lo anterior no se sigue que las criaturas sean parte de Él, sino que hay correspondencia entre las ideas que Él tiene de su creación y su creación misma; es decir, ellas «[aun] cuando no lleguen a asemejarse a Dios por naturaleza con semejanza de especie, como el hombre se asemeja al hombre que le engendra, sin embargo, sí llegan a asemejarse a Él en la representación que de ellas hay en la mente» ${ }^{60}$; es decir, «[...] esta relación de Dios con la criatura no es real, sino sólo de razón ${ }^{61}$. Así, dado que «no es real», el argumento del ens realissimum no tiene cabida. De esto se puede ya concluir algo: no es legítimo predicar algo de Dios solo porque eso también se le puede predicar a las criaturas.

Por otra parte, no es cierto que "existir» no sea un predicado genuino, mientras que «ser» (como verbo) efectivamente no lo es. Esto se debe a que la teoría creacionista tiene una visión trascendental de la naturaleza que, evidentemente, los griegos no tenían.

Un característico principio de los griegos está en creer que la naturaleza ( $\varphi v ́ \sigma \iota \varsigma)$ es completa ${ }^{62}$, autosuficiente, total (ö̉ov) por sí misma; es decir, no es posible pensarse un principio externo (un más allá) ${ }^{63}$ para la naturaleza, como sí pasa con el pensamiento judeocristiano, sino que sus principios, causas y fines les son intrínsecos.

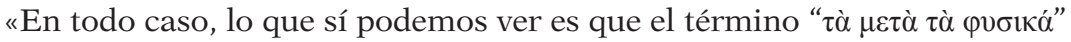
requiere a una idea extraña al pensamiento griego, por lo menos desde los Presocráticos hasta Aristóteles. La cuestión es que para ellos la naturaleza es eterna, es "lo que es", y por lo tanto no hay espacio para "lo que no es" en términos absolutos.» ${ }^{64}$

Por otra parte, la teoría creacionista introduce el concepto «nada» (יאמ/nihil) en II de Macabeos 7:28 para sostener un tipo de acto propio y exclusivo de la comunicación divina ${ }^{65}$. Así, no son comparables los términos «existencia» $\mathrm{y}$ «ser», pues tienen dos fundamentos distintos: el primero, externo; y el segundo, interno. «Existir» sí es un predicado y es significativo ontológicamente ${ }^{66}$, solo

$60 \quad$ Santo Tomás De Aquino, S. T., I, $q .44, a .3, a d .1$

61 Santo Tomás De Aouino, S. T., I, q. 45, a. 3, ad. 1

62 Aristóteles, Met., E, 4, 1015a.

63 Bien lo dice el mismo Aristóteles: «No es posible que haya un solo principio, puesto que los contrarios son una misma cosa [...] pero un principio no puede ser predicado de ningún sujeto, ya que si lo fuera sería el principio de un principio». Fís., I, 6, 189a 10-30; "Además, una cosa puede ser causa de contrarios, pues así como la presencia de una cosa es causa de otra, a veces su ausencia es responsable de su contrario». Ibíd., II, 3, 195a 10.

64 Ortiz Santana, F. E., La filosofía como dialéctica ontológica de los horizontes [tesis de doctorado], Universidad Nacional Autónoma de México, México, D. F. 2014, p. 154

65 Santo Tomás De Aouino, S. T., I, q. 45, a. 5

66 En sentido ontológico, "existir», dentro de la teoría cristiana, sería estar puesto fuera de su raíz, y, por ende, ser/estar incompleto (de ahí la asociación de la existencia y la nada con el vacío y la angustia por parte del existencialismo). En término lógico, «existir» también se toma como un predicado de segundo orden, según el cual se indica: a) que de ese «algo» (un ente) se le pueden predicar cosas (sería un predicado de predicados) ( $\exists \mathrm{X} \Phi \mathrm{X}$ ) (cfr.: FREGE, o. c., § 53; Sobre concepto y objeto, trad. Carlos Ulises Moulines, en: Escritos sobre 
que su relación es de respectividad. El ente es ente en virtud de su tipo de posicionamiento, su valor es tético $0^{67}$, pues «ex-sistere» ${ }^{68}$ lo que indica es que el ente existe en virtud de su posicionamiento fuera de algo; esto es, su raíz: Dios. Así, la relación de las criaturas con Dios es la de estar por fuera de él —lo cual justifica su grado de imperfección-y predicar existencia de ellas significa que están incompletas, que requieren de su raíz (el Bien), y es por esto que todas la buscan llamándola «felicidad».

Ahora bien, de esto no se sigue que el ente sea independiente en sentido absoluto de Dios, sino que su condición ontológica es la de estar por fuera de Él. Al estar fuera de Él, el ente adquiere su valor por respectividad (es creado [ex-siste: está por fuera de su raíz] en virtud de un creador).

\section{3. «El mal ontológico»y «el mal moral»}

Antes que nada, es preciso aclarar que en el párrafo anterior «independiente» y a lo largo de todo el texto «Bien» fue tomando en sentido ontológico (como perfección/ $\dot{v} v \tau \varepsilon \lambda \eta \dot{\chi} \chi \varepsilon l \alpha)$, no en el moral; pues, el hombre, según el cristianismo, sí es libre. Así, queda un último punto a tratar: el problema del mal (II) y el del castigo divino (V), el cual afirma que el castigo de Dios, al ser un mal, es perverso. También es expuesta por Epicuro así:

«- ¿Es que Dios quiere prevenir el mal, pero no es capaz? Entonces no es omnipotente.

- ¿Es capaz, pero no desea hacerlo? Entonces es malévolo.

- ¿Es capaz y desea hacerlo? ¿De dónde surge entonces el mal? Dios?» ${ }^{69}$

- ¿Es que no es capaz ni desea hacerlo? ¿Entonces por qué llamarlo

Respecto a la paradoja II hay que decir lo siguiente: primero, en el caso de las enfermedades o los desastres naturales hay que decir que:

«Algunos, considerando las cosas no en sí mismas, sino por lo que les reportan, las que les resultan nocivas las llaman, sin más, malas. Y no se

lógica, semántica y filosofía de las matemáticas, UNAM, México, D. F. 2016, p. 280; Quine, W. V. O. "On What There Is» in: The Review of Metaphysics, n. 2, 1948, recuperado de: http:// melvinfitting.org/forclasses/phil76600fall2015/quine-on-what-there-is.pdf); y b) que esas cosas que se pueden predicar pueden ser cuantificadas ( $\exists x \varphi x)$ ( $c f r$ : VAN INWAGEN, P. «Metaontology» in: Erkenntnis, n. 48 (1998) 235; Tahko, T. An introduction to Metametaphysics. Cambridge University Press, Cambridge 2015, pp. 15-18).

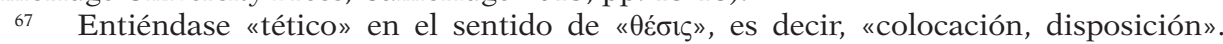
Pabón S., DE Urbina, J. M., Diccionario manual griego-español, quinta edición, Biblograf. S. A., Barcelona 1970, p. 295.

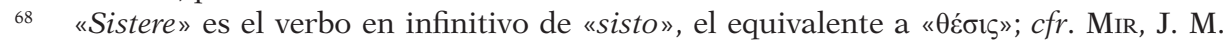
(2002). Diccionario ilustrado latín-español, vigesimoprimera edición, Spes (Vox) Editorial, S. L., Barcelona 2002, voz «sisto». Caso igual pasa con el alemán, donde, de hecho, "Dasein» («existencia») es un verbo separable $(\operatorname{sein}[\ldots] d a)$ que indica «ser/estar ahí».

69 EPICURo, citado por: Hospers, J. An Introduction to Philosophical Analysis, 3rd Edition, Routledge, London, 1990, p. 310. 
detienen a pensar que lo nocivo en algo para unos es beneficioso para otros o también para los mismos en algo. Esto no sería así si las cosas corporales en sí mismas fueran malas y nocivas. ${ }^{70}$

Así, pues, este tipo de hechos no son buenos o malos o por sí mismos, sino que su bondad o maldad se dan por el reconocimiento de los efectos que pueden generar a unos o a otros, sus valoraciones son subjetivas. Como dice el mismo Tomás: "Lo nocivo en algo para unos es beneficioso para otros o también para los mismos en algo ${ }^{71}$. En el caso de los desastres naturales, se valoran hechos físicos como morales, cuando su condición en tanto que hecho es sencillamente natural ${ }^{72}$. En el caso de las enfermedades, puede interpretarse que pueden ser un camino — como hace Dios con Job (38, 1-41) — como una prueba para dirigirse a Él.

Lo segundo que hay que decir a II es que, como ya se dijo antes - dado que la creación no es necesaria y la relación de Dios con sus criaturas no es de pertenencia - que el hombre sea malo — suponiendo que sea el caso $^{73}$ — no indica que Dios sea malo o impotente, pues el origen de la maldad «[...] no es uno determinado y único, sino que cada hombre que no obra rectamente es el verdadero y propio actor de los actos ${ }^{74}$.

Lo anterior resuelve dos cosas: lo primero, el mal no es una cualidad natural de los hombres, pues "La criatura corporal, por naturaleza es buena» ${ }^{75}$; sino que el mal es el alejamiento de la esencia de Dios ${ }^{76}$ : el Bien. El mal, dicho en otros términos, es preferir los bienes contingentes e imperfectos (según Agustín, el hombre) por encima del bien supremo; "[...] no se trata de un bien universal, sino de uno particular y reducido, por lo que se origina en ella [la criatura corporal] cierta contrariedad por la que una se opone a otra aunque en sí mismas sean buenas ${ }^{77}$. De esto se puede inferir lo siguiente: el mismo acto de creación ya es una condición necesaria de posibilidad de la acción, pues es necesario de facto elegir entre un bien imperfecto u otro, o, dicho de una mejor

70 Santo Tomás De Aquino, S. T., I, q. 65, a. 1, ad. 2.

71 Santo Tomís De Aquino, S. T., I, $q .65, a .1, a d ., 2$.

72 Es decir, los hechos como tal no son buenos ni malos, sino que el valor moral está en el reconocimiento (según posturas actuales, desde el lenguaje) de dichos hechos como buenos o malos. Así, «El asesinato estará en el mismo nivel que cualquier otro acontecimiento como, por ejemplo, la caída de una piedra. Ciertamente, la lectura de esta descripción puede causarnos dolor o rabia o cualquier otra emoción; también podríamos leer acerca del dolor o la rabia que este asesinato ha suscitado entre otra gente que tuvo conocimiento de él, pero serían implemente hechos, hechos y hechos, y no ética». Wittgenstein, Conferencia sobre ética, trad. Fina Birulés, en: Reguera, I. (ed.), Obra completa, vol. 2, Gredos, Biblioteca de Grandes Pensadores, Madrid, p. 518.

73 De hecho, «Ninguna criatura de Dios es mala, sino que el mal consiste en hacer mal uso de ella» (SAn Agustín, 1947c, XXXVI).

$74 \quad$ San Agustín, Del libre albedrío, I, $1, \S 1$.

75 Santo Tomás De Aquino, S. T., I, $q .65, a .1, a d .2$.

76 «En consecuencia, nuestro pecado es deprecio del Creador. Y pecar es despreciar al Creador, es decir, no hacer por Él lo que creemos que debemos hacer» (Abelardo, 1994, c. 3).

77 Santo Tomás De Aquino, loc. cit. 
manera: entre quedarse en el bien imperfecto y particular o ascender a partir de este hasta el perfecto y universal; pues que las criaturas corporales «[...] alejen de Dios se debe a aquellos que hacen un torpe uso de ellas ${ }^{78}$.

Pasemos, por último, a la paradoja V: los dos primeros cuestionamientos de Epicuro $^{79}$ remiten forzosamente a cuestionar el papel de Dios en el gobierno del mundo: ¿debe intervenir en las acciones humanas para evitar que el hombre haga el mal, es decir, se aleje de Él?

Ante estos cuestionamientos vale decir lo siguiente: primero, dado que Dios solo puede hacer el Bien, entonces el acto de creación es por sí mismo perfecto $^{80}$, es decir, "Dios hace todo de la manera más deseable» ${ }^{81}$ este es el mejor de los mundos posibles ${ }^{82}$.

Si es cierto que la creación divina implica la generación de una degradación del bien en las criaturas, y, que precisamente por esto, de facto los hombres están obligados a escoger libremente entre el bien particular y el Bien de Dios, entonces la condición de la libertad divina es la mejor de las condiciones posibles. Así, entre un determinismo absolutista en el cual Dios coarta la acción humana o que el hombre sea libre, la segunda opción es la mejor posible.

Además de la condición de facto, también es preciso afirmar que la misma naturaleza racional del hombre es condición necesaria de la libertad del mismo, que es un don ofrecido por Dios para que este (el hombre) alcanzarle a Él, que es la suma bondad ${ }^{83}$. Con esto, el «es» (la condición natural del hombre) contiene implícitamente al «debe» por tener la facultad de poder reconocerlo ${ }^{84}$. Además, el hombre reconoce el deber de sus acciones al valorar, por medio de su razón, a los entes y efectos en su grado de perfección, bondad o felicidad que este le puede proporcionar. Así, por naturaleza Dios indica y le posibilita al sujeto moral el reconocimiento del deber a partir del don de la libertad.

78 Santo Tomás De Aouino, S. T., I, q. 65, a. 1, ad. 3.

79 El tercero es resuelto por lo anteriormente explicado en la respuesta a la paradoja II. El cuarto es sencillamente contradictorio, pues, como ya se dijo antes, Dios es omnipotente y bueno.

${ }_{80}$ Hay que tener cuidado con esto: el acto mismo de creación es el perfecto, no la criatura misma.

81 LeIBNIz, o. c., Tratado de metafísica, § 1.

82 Leibniz, o. c., Profesión de fe del filósofo, p. 52.

83 Santo Tomás De Aquino, S. T., I, $q .103$.

84 Esto es conocido como «el problema del paso del es al debe», donde se intenta explicar cómo se reconoce el deber de manera natural —es decir, cómo el «debe» está inmerso en el «es»—, sin caer en la falacia naturalista ( $c f r$. Platón, La República, trad. José Pabón y Manuel Fernández-Galiano, edición bilingüe. Centro de Estudios Políticos y Constitucionales, Madrid 2006, I, 338c-353c). Algunos estudiosos de la filosofía analítica, como Rodríguez siguiendo a Searle, han planteado alternativas de solución desde el lenguaje y los actos del habla; $c f r$.: Rodríguez Ortiz, A. M. "Searle y la posibilidad de derivar un "debe" de un "es" " en: Escritos, n. 23, vol. 50 (2015), recuperado de: https://revistas.upb.edu.co/index.php/escritos/ article/download/4552/4108. Esta postura, sin embargo, se ampara en elementos ontológicos (factuales) y gnoseológicos (cognitivos) amparados en una perspectiva metafísica. Así, parece que la propuesta tomista y cristiana permite solucionar problemas «contemporáneos» sobre la moral, su fundamento y sus condiciones necesarias de posibilidad. 
Pero, entonces, ¿Dios no gobierna al mundo? ¿Cómo interviene Dios en el mundo y en las acciones humanas? Efectivamente Dios gobierna al mundo ${ }^{85}$, solo que lo hace por dos vías: primero, por el castigo, segundo, por las enseñanzas.

A primera vista, el primer medio de Dios es distinto al segundo; ejecutar un diluvio universal parece ser mucho más violento que lo que sería; sin embargo, la finalidad de sus castigos es, a través de la disciplina, comunicarnos la verdad y el camino hacia el Bien ${ }^{86}$. Así, pues, Dios interviene en el mundo a partir de la enseñanza, como un maestro, guiando a las criaturas para que se acerquen a Él ${ }^{87}$. Por esto, no es cierto que Él no quiere que se erradique el mal en el mundo.

\section{CONCLUSIONES}

Una vez resueltas las paradojas, se pueden concluir las siguientes cosas: 1 . La necesidad de Dios le es interna; la externa no le afecta en sentido alguno, pues la segunda parte de un supuesto y su necesidad es relativa; 2 . Dios es omnipotente y, dada la definición de omnipotente como «aquello que puede hacer todo lo posible», solo puede hacer lo que es posible en sentido absoluto; 3. La relación de Dios con las criaturas es de comunicación y no de necesidad o participación, pues el acto creador no le es necesario esencialmente a Dios, sino que lo es por relación; 4. La libertad de Dios ni la del hombre atentan contra la omnipotencia divina, pues: $\alpha$ ) la creación no le es necesaria esencialmente a Dios; y $\beta$ ) si la libertad es producto de la creación, entonces es la mejor de las situaciones posibles: dar la oportunidad al hombre de actuar bien por su propia voluntad para alcanzarle a É $\mathbf{l}^{88}$ y al cielo; 5 . Es preferible la intervención divina aceptando la libertad y la enseñanza que un determinismo coercitivo por parte de Él (por $\beta)$. Teniendo en cuenta esto, se puede concluir que las principales paradojas presentadas hasta el momento carecen de fundamentos, dada su incomprensión de los argumentos, conceptos y raciocinios por parte de los cristianos. Así, en caso de querer establecer un debate genuino entre ambas partes, es necesario por parte de los ateístas comprender y saber criticar estas respuestas desde su raíz, y, por otra parte, es preciso que los cristianos y creyentes estén dispuestos a seguir reelaborando sus respuestas de tal forma que la discusión se enriquezca y el debate como tal se siga ampliando cada vez más.

[Artículo aprobado para publicación en enero de 2018]

\footnotetext{
85 Santo Tomás De Aquino, S. T., I, q. 103, a. 1.

86 SAN Agustín, ibíd.

87 Santo Tomás De Aquino, S. T., I, q. 83, a. 1, sol.

88 LEIBNIZ, O. c., p. 88.
} 